\title{
Phenotypic Identification and Antifungal Susceptibility of Two Rhodotorula Species Isolated from Dandruff Samples
}

\author{
Kohinur Begum, Mashmum Shababa Shahid, Sanzida Ferdoush \\ and Farjahan Nur
}

\author{
Department of Pharmacy, ASA University Bangladesh, ASA Tower, Shyamoli, Dhaka-1207 Bangladesh
}

(Received: November 03, 2020; Accepted: November 28, 2020; Published (web): December 28, 2020)

\begin{abstract}
Rhodotorula are environmental yeasts originally considered non-pathogenic in nature. However, over the last three decades, different species of this yeast have established themselves as pathogens in humans, causing systemic infections among the immunocompromised population. In this study, Rhodotorula species were isolated from dandruff samples using selective media by observing colony morphology and color. The isolates were later identified via biochemical tests and microscopic examinations. In addition, the sensitivity of the isolates to the three antifungal agents, namely ketoconazole, nystatin and fluconazole were tested by using the disk diffusion technique. On completion of the tests, only two species of Rhodotorula were identified from 35 dandruff samples and designated as R1 and R3. Both isolates displayed sensitivity towards ketoconazole and nystatin. No antifungal sensitivity was documented against fluconazole. This study gave preliminary indication of the presence of Rhodotorula species in dandruff samples, and its sensitivity towards antifungal agents.
\end{abstract}

Key words: Rhodotorula, Dandruff, Antifungal activity.

\section{INTRODUCTION}

Rhodotorula species are saprophytic yeasts, ubiquitous in nature mostly as non-pathogenic strains. However, the species $R$. mucilaginosa, $R$. glutinis, and $R$. minuta of the genus have yet been documented to cause diseases in humans. ${ }^{1}$ Only a little scientific details can be found regarding the epidemiology, risk factors and outcomes of Rhodotorula-related mycoses in humans. ${ }^{2-4}$ Recent findings have identified Rhodotorula spp. as emerging opportunistic pathogens with the capacity to colonize and infect immunocompromised patients causing catheter-associated fungaemia, onychomycosis, meningitis and peritonitis dermatitis, oral ulcers, aortic homograft endocarditis and lymphadenitis. ${ }^{5-10}$ Majority of these systemic infections are treated using antifungal agents such as amphotericin B and azoles viz. ketoconazole, fluconazole etc. ${ }^{11}$

Correspondence to: Kohinur Begum

E-mail: kohinur025@yahoo.com, prof.kohinur@asaub.edu.bd

Dhaka Univ. J. Pharm. Sci. 19(2): 185-190, 2020 (December) DOI: https://doi.org/10.3329/dujps.v19i2.50635
Unlike the systemic infections, dandruff is a common scalp condition targeting people of all ages and health status. ${ }^{12-14}$ The genus Malassezia is commonly associated with dandruff as opposed to the genus Rhodotorula. ${ }^{15,16}$ Nevertheless, Rhodotorula mucilaginosa has been detected on healthy scalps ${ }^{17}$ and reported as part of skin microbiota responsible for atopic dermatitis in humans. ${ }^{18}$ Hence the possibility of Rhodotorula spp. being causative agents of dandruff is not completely improbable. Therefore, the objective of this study was to isolate and characterize species of Rhodotorula from dandruff samples and determine the efficacy of the three antifungal agents including ketoconazole, nystatin and fluconazole against it.

\section{MATERIALS AND METHODS}

Material. Antifungal drugs which are ketoconazole, nystatin and fluconazole were provided by Incepta Pharmaceuticals Ltd. All other chemicals and reagents used were of analytical grade.

Sample collection. A total of 35 samples were collected from 20 male and 15 female students of 
ASA University Bangladesh, ranging from 18 to 24 years of age. The dandruff flakes were collected from the scalp by combing using a sterile comb within the period of the year 2018 and 2019.

Culture and isolation of colonies. All samples were enriched by inoculating in $5 \mathrm{ml}$ Sabouraud dextrose broth (HiMedia, India) and incubated overnight with gentle shaking at $30^{\circ} \mathrm{C}$. Enriched cultures were streaked on Sabouraud dextrose agar (SDA) containing chloramphenicol $(0.05 \%)$ and incubated at $30^{\circ} \mathrm{C}$ for three days. The suspected single colonies were isolated by observing colony morphology and color. The isolates of Rhodotorula spp. were screened based on the texture and typical color exhibited by their colonies on SDA, as well as by their micromorphology. Rhodotorula spp. were able to produce carotenoid pigments conferring a salmon-pink to coral-red color to the colonies. Microscopic examination was performed by standard Gram staining to determine the cell shape and type of budding. Only spheroidal to oval budding cells without the rudimentary formation of hyphae were considered as these belong to the genus Rhodotorula. $^{19,20}$ Isolates were stocked in yeast peptone dextrose (YPD) media with $30 \%$ glycerol in $-20^{\circ} \mathrm{C}$

Biochemical tests. Following preliminary identification, the isolates were further characterized by biochemical tests such as catalase test, oxidase test, bile esculin test, sugar and nitrate assimilation tests and survivability in different temperatures.

Catalase test. The catalase test was done to detect the presence of catalase enzyme having ability to break hydrogen peroxide to release oxygen and water. The catalase test was performed by using a drop of 3\% hydrogen peroxide placed on glass slide. A freshly prepared colony was transferred with loop and mixed well. The production of gas bubbles indicated catalase positive and the absence as catalase negative. C. albicans was used as the positive control.

Oxidase test. The oxidase test was performed to detect the presence of the terminal enzyme cytochrome $\mathrm{C}$ oxidase according to standard oxidase test protocol. $1 \% \quad \mathrm{~N}, \mathrm{~N}, \mathrm{~N}^{\prime}, \mathrm{N}^{\prime}$-tetramethyl-p-phenylenediamine (TMPD) solution was used as oxidizing agent. A freshly prepared isolated colony was placed with a platinum loop onto filter paper soaked with TMPD. Result was recorded by observing color change to deep blue or purple. Dark purple color appeared within 10 to 30 seconds and indicated oxidase positive and no color change after 2 minutes indicated negative response for oxidase test. $C$. albicans was used as negative control.

Bile esculin test. Bile-esculin test was performed by using bile-esculin agar slants. Freshly prepared colonies were inoculated onto the slant with an S-shaped motion, and incubated at $30^{\circ} \mathrm{C}$ for 3 days. Slant without inoculation was used as control. Positive results indicated growth and blackening of the media compared with the control, while growth but no blackening of medium was counted as negative result. $C$. albicans was used as the negative control. All tests were done in duplicate.

Nitrate assimilation test. Modified nitrate assimilation agar test was performed using yeast nitrogen base media (YNB, Sigma-Aldrich) with potassium nitrate and bromothymol blue agar slants. Isolates were inoculated onto agar slants and incubated at $30^{\circ} \mathrm{C}$ for 3 days. Potassium nitrate provided the nitrogen source and bromothymol blue as $\mathrm{pH}$ indicator that changes from green to blue causing nitrogen utilization. C. albicans was used for the positive control. Slant without inoculation was used as the negative control. Results were recorded by observing color change compared with control. Each test was done in duplicate.

Sugar assimilation test. Sugar assimilation test was performed by using YNB, with $1 \%$ sugar such as glucose, sucrose, fructose, lactose and xylose in agar slants. Freshly prepared isolates were inoculated and incubated at $30^{\circ} \mathrm{C}$ for 3 days. A positive reaction was indicated by growth on the slant and change in color from purple to yellow, while no color changes of medium was considered as negative result. C. albicans was used as the positive control. Slant without inoculation was used as the negative control. Each test was performed in duplicate. 
Effect of temperature on the growth. Effects of temperature on isolates were determined in extreme temperature conditions. Isolates were inoculated on SDA plates and incubated at $5^{\circ} \mathrm{C}, 40^{\circ} \mathrm{C}$, $45^{\circ} \mathrm{C}, 50^{\circ} \mathrm{C}, 55^{\circ} \mathrm{C}$ and $60^{\circ} \mathrm{C}$ for 3 days. Results were recorded every 24 hours for 3 days. The growth of colonies indicated the positive result. No growth was recorded as the negative result. Inoculation with $C$. albicans and without culture plates were used as the positive and the negative control, respectively.

Antifungal assay. Antifungal assay was performed by disk diffusion method using SDA agar. ${ }^{21}$ Ketoconazole, nystatin and fluconazole were used as antifungal drugs. Cell suspension $\left(10^{5}\right.$ cells $/ \mathrm{ml}$ ) was spread uniformly on agar plates. Each disk containing $50 \mu \mathrm{g}$ drug was placed on the surface of isolate spreading SDA plate. The SDA plates were incubated in the upright position at $30^{\circ} \mathrm{C}$ for 3 days. Disk containing DMSO was used as the negative control. C. albicans was used as the positive control strain. Diameter of the zone of inhibition was recorded in millimeters and average was taken from the duplicate tests.

\section{RESULTS AND DISCUSSION}

Fungal microbiota associated with dandruff and other dermatological infections can pose a threat to human health. ${ }^{22}$ Only a few studies have been reported to decipher the fungal microbiota in dandruff. From this particular study, two species of Rhodotorula, designated as R1 and R3 have been successfully isolated and identified from 35 dandruff samples by observing the colony color and morphology on SDA plate. The distinctive coral-red colony was found due to the presence of carotenoid pigments along with smooth, glistening and mucoid appearance on the agar plate as shown in figure 1 and the oval, budding cells were also found under microscopic observation in figure 2. Rhodotorula spp. varies from other yeasts by producing pink-tored colonies and lacking pseudohyphae.

In this study, Rhodotorula spp. were identified by observing results of biochemical tests as depicted in Table 1 and results were comparable with the control. By changing the color of media and showing growth, two Rhodotorula spp. were found positive for nitrate and sugar assimilation test as shown in Table 2. Positive results of sugar and nitrate assimilation tests were comparable to the expected patterns of Rhodotorula spp. in other studies. Yeasts have their ability to utilize carbohydrates for energy, both aerobically (assimilation) and in some cases anaerobically (fermentation). The patterns of carbohydrates assimilation and fermentation are characteristic of the different yeast species, as well as identifying characteristics of unknown yeast isolates.

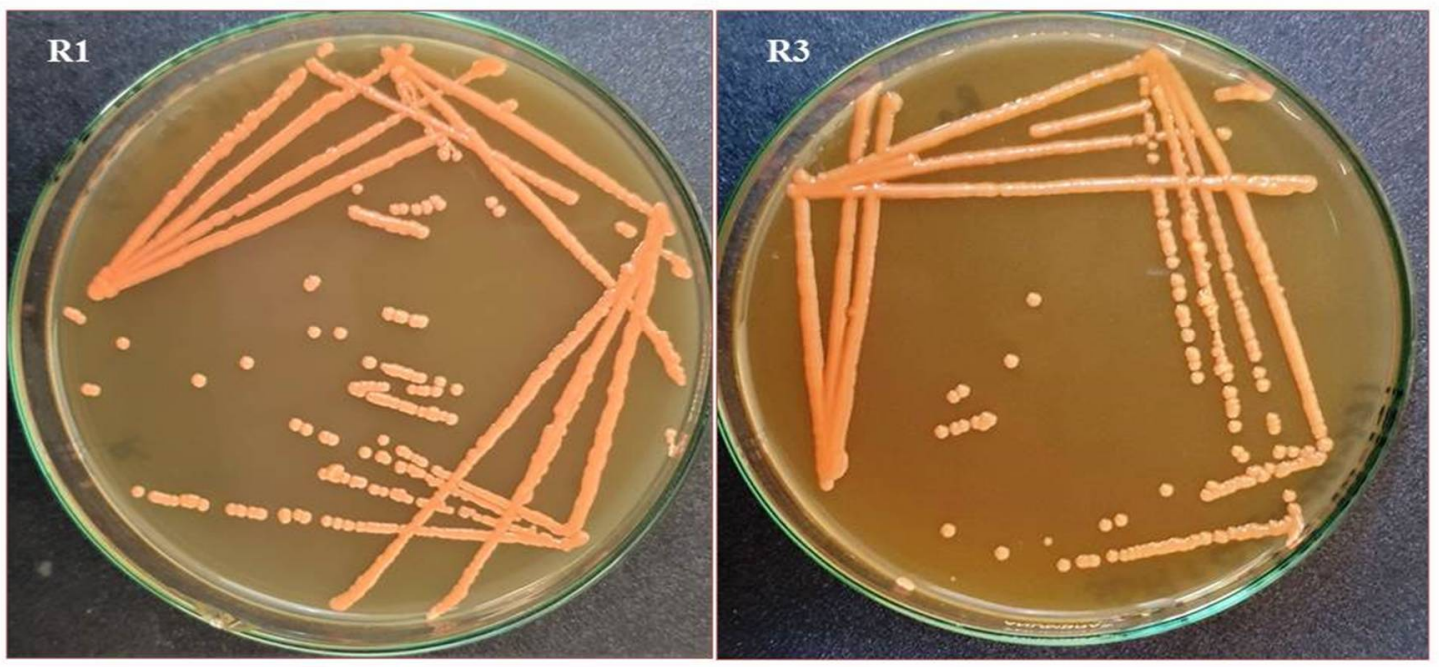

Figure 1. Colony morphology of Rhodotorula species (R1 and R3) on SDA plate. 


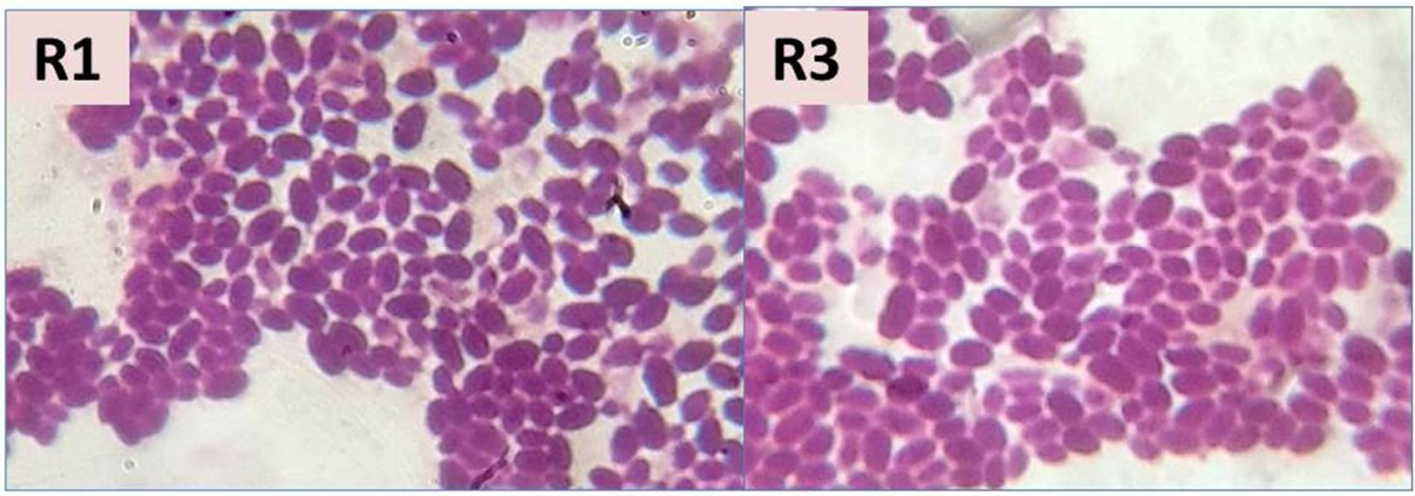

Figure 2. Microscopic observation of Rhodotorula species (R1 and R3) by Gram Staining.

Table 1. Biochemical tests of isolated Rhodotorula species.

\begin{tabular}{|c|c|c|c|c|c|c|c|c|c|}
\hline \multirow[t]{2}{*}{ Isolate name } & \multirow[t]{2}{*}{ Catalase test } & \multirow{2}{*}{$\begin{array}{c}\text { Oxidase } \\
\text { test }\end{array}$} & \multirow{2}{*}{$\begin{array}{l}\text { Bile esculin } \\
\text { test }\end{array}$} & \multicolumn{6}{|c|}{ Temperature } \\
\hline & & & & $5^{\circ} \mathrm{C}$ & $40^{\circ} \mathrm{C}$ & $45^{\circ} \mathrm{C}$ & $50^{\circ} \mathrm{C}$ & $55^{\circ} \mathrm{C}$ & $60^{\circ} \mathrm{C}$ \\
\hline R1 & - & - & + & + & + & + & + & + & - \\
\hline R3 & - & - & + & + & + & + & + & + & - \\
\hline $\begin{array}{l}\text { C. albicans } \\
\text { (Control) }\end{array}$ & + & - & - & + & + & + & - & - & - \\
\hline
\end{tabular}

$+=$ positive result/growth, $-=$ negative result/no growth

Table 2. Sugar and nitrate assimilation test of Rhodotorula species.

\begin{tabular}{lcccccc}
\hline Isolate name & \multicolumn{7}{c}{ Carbohydrate tests } & \multirow{2}{*}{ Nitrate test } \\
\cline { 2 - 6 } & Glucose & Sucrose & Fructose & Lactose & Xylose & \\
\hline R1 & + & + & + & + & + & + \\
R3 & + & + & + & + & + & + \\
C. albicans & + & + & + & + & + & + \\
(Control) & & & & & & \\
\hline
\end{tabular}

$+=$ positive result.
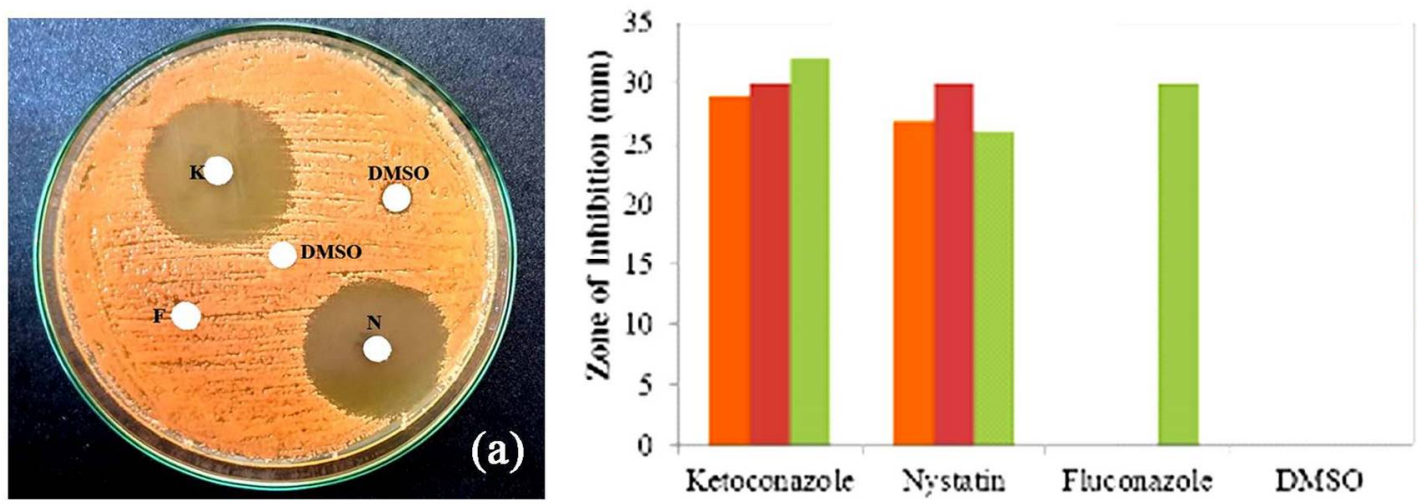

$=\mathrm{R} !$

$=\mathrm{R} \cdot 3$

Exanticla

allsicatos

(b)

Figure 3. Antifungal susceptibility of Rhodotorula spp. a. plate showing sensitivity of the strain R3 to ketoconazole (K), fluconazole (F), nystatin (N) and DMSO, and b. corresponding zone of inhibition for both strain R1 and R3. 
Temperature effect is another characteristic property of yeast isolation. Rhodotorula spp. can survive at temperatures ranging from $5^{\circ} \mathrm{C}$ to $42^{\circ} \mathrm{C}$ and optimal temperature between $20^{\circ} \mathrm{C}$ and $25^{\circ} \mathrm{C}$. In our study, Rhodotorula spp. was found to grow optimally at $30^{\circ} \mathrm{C}$ and even survived at the extremes of $5^{\circ} \mathrm{C}$ and $55^{\circ} \mathrm{C}$, as shown in Table 1. Variation of these results may be attributed to diverse sources of isolation. Availability of fungi yeast in the environment varies among geographic areas, and its distribution is influenced by several factors such as temperature, humidity, seasonal variation and human activities. ${ }^{23}$

A few available data in the literature have been reported on the isolation and antifungal susceptibility of this yeast. Antifungal agents such as 5fluorocytosine, amphotericin B, nystatin and azoles have found to be effective against clinically isolated Rhodotorula spp. A study ${ }^{24}$ tested 35 clinically isolated Rhodotorula strains against several antifungal agents and found that all the strains were sensitive to 5-fluorocytosine, amphotericin B, ketoconazole and itraconazole but resistant to fluconazole. In this study, both R1 and R3 strains were found sensitive to ketoconazole, and nystatin exhibiting zone of inhibition (> $27 \mathrm{~mm}$ ) but fluconazole did not give zone of inhibition and shown in figure 3. Therefore, our result is consistent with others antifungal sensitivity studies performed in vitro against Rhodotorula.

\section{CONCLUSIONS}

This study demonstrated that Rhodotorula species are present in dandruff in human scalp. Furthermore, these Rhodotorula species showed sensitivity toward ketoconazole and nystatin, two antifungal drugs. However, fluconazole was found to be inactive against Rhodotorula. From this study, it can be concluded that Rhodotorula spp. with other microbiota may contribute to serious dandruff problems in human. Further an extensive research is required to identify species level involving mass population and the development of effective antifungal agents for combating dandruff.

\section{REFERENCES}

1. Larone, D. H. 1995. Medically Important Fungi: A Guide to Identification. 3rd ed., ASM Press, Washington (D.C.), p. 274.

2. Duboc De Almeida, G.M., Costa, S.F., Melhem, M., Motta, A.L., Walderez Szeszs, M., Miyashita, F., Pierrotti, L.C., Rossi, F. and Burattini, M.N. 2008. Rhodotorula spp. isolated from blood cultures: clinical and microbiological aspects. Med. Mycol. 46, 547-556.

3. Tuon, F.F., Duboc de Almeida, G.M. and Costa, S.F. 2007. Central venous catheter-associated fungemia due to Rhodotorula spp.-a systematic review. Med. Mycol. 45, 441447.

4. Tuon, F.F. and Costa, S.F. 2008. Rhodotorula infection. A systematic review of 128 cases from literature. Rev. Iberoam. Micol. 25, 135-140.

5. Lunardi, L.W., Aquino, V.R., Zimerman, R.A. and Goldani, L.Z. 2006. Epidemiology and outcome of Rhodotorula fungemia in a tertiary care hospital. Clin. Infect. Dis. 43, e60e63.

6. Kaur, R., Wadhwa, A. and Agarwal, S.K. 2007. Rhodotorula mucilaginosa: an unusual cause of oral ulcers in AIDS patients. AIDS. 21, 1068-1069.

7. Means, A.D., Sisto, K., Lichon, V., Monaghan, D., O'Keefe, P. and Tung, R. 2012. Cutaneous rhodotorula treated with photodynamic therapy. Dermatol. Surg. 38, 1100-1103.

8. Maeder, M., Vogt, P.R., Schaer, G., Von Graevenitz, A. and Günthard, H.F. 2003. Aortic homograft endocarditis caused by Rhodotorula mucilaginosa. Infection. 31, 181-183.

9. Fung, H.B., Martyn, C.A., Shahidi, A. and Brown, S.T. 2009. Rhodotorula mucilaginosa lymphadenitis in an HIV-infected patient. Int. J. Infect. Dis. 13, e27-e29.

10. Fell, J.W., Boekhout, T., Fonseca, A., Scorzetti, G. and Statzell-Tallman, A. 2000. Biodiversity and systematics of basidiomycetous yeasts as determined by large-subunit rDNA D1/D2 domain sequence analysis. Int. J. Syst. Evol. Microbiol. 50, 1351-1371.

11. Diekema, D.J., Petroelje, B., Messer, S.A., Hollis, R.J. and Pfaller, M.A. 2005. Activities of available and investigational antifungal agents against Rhodotorula species. J. Clin. Microbiol. 43, 476-478.

12. Ranganathan, S. and Mukhopadhyay, T. 2010. Dandruff: The most commercially exploited skin disease. Indian $J$. Dermatol. 55, 130-134.

13. Ro, B.I. and Dawson, T.L. 2005. The role of sebaceous gland activity and scalp microfloral metabolism in the etiology of seborrheic dermatitis and dandruff. J. Investig. Dermatol. Symp. Proc. 10, 194-197.

14. Piérard-Franchimont, C., Xhauflaire-Uhoda, E. and Piérard, G.E. 2006. Revisiting dandruff. Int. J. Cosmet. Sci. 28, $311-$ 318. 
15. Gupta, A.K., Madzia, S.E. and Batra, R. 2004. Etiology and management of seborrheic dermatitis. Dermatology. 208, 89-93.

16. Begum, K., Nur, F. and Shahid, M.S. 2019. Isolation and characterization of Malasezzia species from dandruff samples and determination of its sensitivity towards antifungal agents. Bangladesh Pharm. J. 22, 146-152.

17. Park, H.K., Ha, M.H., Park, S.G., Kim, M.N., Kim, B.J. and Kim, W. 2012. Characterization of the fungal microbiota (mycobiome) in healthy and dandruff-afflicted human scalps. PloS ONE, 7, e32847.

18. Zinkeviciene, A., Vaiciulioniene, N., Baranauskiene, I., Kvedariene, V., Emuzyte, R. and Citavicius, D. 2011. Cutaneous yeast microflora in patients with atopic dermatitis. Cent. Eur. J. Med. 6, 713-719.

19. de Hoog G.S., Guarro J., Gené J. and Figueras M.J. 2000. Atlas of Clinical Fungi. 2nd ed., Centraalbureau voor Schimmelcultures, Utrecht, The Netherlands, p.1126.
20. Statzell-Tallman, A. and Fell, J.W. 1998. Rhodotorula FC Harrison. In: The Yeasts: A Taxonomic Study (Kurtzman, C.P. and Fell J.W., Eds.), 4th ed., Elsevier, New York, pp. 800-827.

21. Bauer, A.W., Kirby, W.M.M., Sherris, J.C. and Turck, M. 1966. Antibiotic susceptibility testing by a standardized single disc method. Am. J. Clin. Pathol. 36, 493-496.

22. Butler, G. 2010. Fungal sex and pathogenesis. Clin. Microbiol. Rev. 23, 140-159.

23. Nagahama, T., Hamamoto, M., Nakase, T. and Horikoshi, K. 2001. Rhodotorula lamellibrachii sp. nov., a new yeast species from a tubeworm collected at the deep-sea floor in Sagami Bay and its phylogenetic analysis. Antonie van Leeuwenhoek, 80, 317-323.

24. Galan-Sanchez F., Garcia-Martos, P., Rodriguez-Ramos, C., Marın-Casanova, P., Mira-Gutierrez, G. 1999. Microbiological characteristics and susceptibility patterns of strains of Rhodotorula isolated from clinical samples. Mycopathologia, 145, 109-112. 